\title{
Meticillin-resistant Staphylococcus aureus (MRSA) bacteraemia in Tampere University Hospital: a case- control study, Finland October 2002 to January 2010
}

P Rahikka1, J Syrjänen ${ }^{1,2}$, R Vuento ${ }^{3}$, J Laine ${ }^{2}$, R Huttunen (reetta.huttunen@uta.fi) ${ }^{1,2}$

1. University of Tampere Medical School, Fl-33014 University of Tampere, Finland

2. Department of Internal Medicine, Tampere University Hospital, Tampere, Finland

3. Centre for Laboratory Medicine, Pirkanmaa Hospital District, Tampere, Finland

Citation style for this article:

Rahikka P, Syrjänen J, Vuento R, Laine J, Huttunen R. Meticillin-resistant Staphylococcus aureus (MRSA) bacteraemia in Tampere University Hospital: a case-control study, Finland October 2002 to January 2010

Euro Surveill. 2011;16(35):pii=19958. Available online: http://www.eurosurveillance.org/ViewArticle.aspx?Articleld=19958

Article published on 1 September 2011

Meticillin-resistant Staphylococcus aureus (MRSA) has emerged as a frequent pathogen in blood cultures in Pirkanmaa Hospital District (HD), Finland. To study risk factors for MRSA bacteraemia and the adequacy of empirical antimicrobial treatment, we retrospectively reviewed the hospital records of 102 patients, 51 with MRSA, and 51 with meticillin-sensitive Staphylococcus aureus (MSSA) bacteraemias respectively, who had been admitted to Tampere University Hospital in Pirkanmaa HD, from October 2002 to January 2010. For each patient with MRSA bacteraemia, one consecutively detected unmatched patient with MSSA bacteraemia was chosen as control. Patients with MRSA bacteraemias were significantly older (median age: 73 years vs 59 years, $p=0.001$ ), were more likely to have been transferred directly from another healthcare facility or were already in the hospital at the onset of bacteraemia (39/51 vs 26/51, $p=0.007$ ) and had a higher McCabe class than patients with MSSA bacteraemia $(p=0.005)$. Patients with MRSA bacteraemia more seldom received adequate empirical antimicrobial therapy when compared to those with MSSA bacteraemia (13/51 vs 43/51, p<0.001). Of previously known MRSA carriers 10 of 29 received adequate empirical antimicrobial therapy for their condition. The percentage of MRSA bacteraemias among all $S$. aureus bacteraemias in Pirkanmaa HD is high compared to corresponding figures for the whole of Finland.

\section{Introduction}

Meticillin-resistant Staphyloccus aureus (MRSA) is a significant cause of both healthcare- and communityacquired infections [1]. The spread of MRSA in hospitals and healthcare facilities is a major threat, mainly for patients with underlying conditions. Studies conducted in countries with endemic MRSA have shown that patients with MRSA bacteraemia have more comorbidities and are significantly older than those with meticillin-sensitive S. aureus (MSSA) bacteraemia [2-4]. The worldwide emergence of MRSA-associated infections has prompted an Expert Panel of the Infectious
Diseases Society of America to publish evidence-based guidelines for the management of patients with MRSA infections [1].

MRSA has emerged as a frequent pathogen isolated in severe infections such as bacteraemia, in regions previously reported to be low-prevalence areas, for example Sweden and Finland [5,6]. From 1997 to 2003, the annual number of MRSA notifications to the National Infectious Disease Register in Finland rose over tenfold, and the proportion of MRSA among S. aureus blood isolates tripled, from less than $1 \%$ to $2.8 \%$ in 2004 [7].

In the Pirkanmaa Hospital District (HD), Finland, the number of new MRSA notifications has increased during the past 10 years and MRSA bacteraemia has emerged as a prevalent pathogen isolated in blood cultures. MRSA epidemics began in Pirkanmaa HD in 2001 [unpublished data]. Since then, Pirkanmaa HD has conducted routine surveillance for MRSA. From 2001 to 2007, Pirkanmaa HD recommended that patients admitted in Tampere University Hospital (TAUH), the central hospital of the Pirkanmaa HD, should be screened for MRSA if they had been treated in long-term care facilities of Tampere or in high-MRSA-incidence wards in Tampere city hospitals. During recent years, from 2008 to July 2011, Pirkanmaa HD recommended that all patients admitted to hospitals/healthcare institutions should be screened for MRSA if they had been treated in any hospital, healthcare institution or long-term care unit of the Pirkanmaa HD after 2001. Since August 2011, universal screening of all patients admitted to hospitals/healthcare institutions of the Pirkanmaa HD is conducted.

We here carried out a retrospective case-control study in TAUH, Finland, to compare underlying patient characteristics and the adequacy of empirical therapy in MSSA and MRSA bacteraemias. 


\section{Methods}

\section{Pirkanmaa Hospital District}

The Pirkanmaa HD is a joint municipal authority comprising 22 municipalities and responsible for healthcare services to about 477,600 inhabitants (population in year 2009). The overall population of Finland in 2009 was $5,351,427$. Thus, Pirkanmaa HD provides healthcare for $9 \%$ of the total Finnish population [8].

\section{Study population}

The only central hospital in the Pirkanmaa HD area is TAUH. The present study comprised 51 patients with blood culture-confirmed MRSA bacteraemia and 51 unmatched control patients with MSSA confirmed bacteraemia admitted to TAUH, from October 2002 to January 2010. All MRSA bacteraemia patients ever treated in TAUH were included. Patients with polymicrobial bacteraemia were excluded (three cases) and only the first bacteraemia episode per patient, for those with multiple episodes was included. For each patient with MRSA bacteraemia a consecutively treated unmatched patient with MSSA bacteraemia was selected as control. Underlying diseases, clinical data and laboratory parameters were retrospectively reviewed from the hospital records. Patients were defined as previously known MRSA carriers, if their patient history indicated that MRSA had been detected by screening or in clinical samples prior to bacteraemia suspicion. Information regarding a previous MRSA finding was available in the patients hospital records. Chronic diseases and sources of bacteraemia were registered. Alcohol-abuse was defined as a known social or medical problem due to alcohol use. Patients were defined as current smokers and non-smokers, i.e. those who had never smoked or had stopped smoking. McCabe classification [9] was used to determine the severity of any underlying disease.

\section{Diagnostics}

In our hospital blood cultures are routinely taken in cases with symptoms or signs of systemic infection. To obtain blood for culture, two sequential samples of blood for anaerobic and aerobic culture $(2 \times(20+20 \mathrm{ml}))$ were taken through venipuncture according to the manufacturer's instructions. The BACTEC 9240 (BD Diagnostic Systems, Sparks, MD, USA) blood culture system was used with standard media until November 2006, and the BactALERT (BacT/ALERT 3D (bioMérieux, Marcy l'Etoile, France) was used from December 2006.

\section{Case fatality}

The case fatality rate was studied within 30 days after a positive blood culture.

\section{Antimicrobial treatment}

Empirical antimicrobial treatment for MRSA was considered adequate when (i) the treatment was started or continued on the day or the day after ( +1 day) the blood culture was taken, and (ii) when it was applied intravenously (with the exception of linezolid, which was considered adequate also when taken perorally) and (iii) contained at least one element with good activity against the MRSA strain in question (vancomycin, linezolid, daptomycin or teicoplanin). Intravenous clindamycin was only considered adequate when the S. aureus strain was susceptible to both clindamycin and erythromycin. Therapy against MSSA was considered adequate if it contained at least one intravenous regimen with good activity against MSSA. For one patient with MSSA bacteraemia receiving levofloxacin, which has a good bioavailability orally as an empiric regimen, the therapy was considered adequate.

\section{Statistical analysis}

An SPSS package (version 10.0) was used for statistical analyses and a two-sided p-value $<0.05$ was taken as cut-off for statistical significance. Categorical data were analyzed by chi-square test or Fisher's exact test when appropriate and nonparametric data by MannWhitney U-test. Odds ratios (ORs) were expressed with their $95 \%$ confidence intervals $(\mathrm{Cl})$.

\section{Results}

The total number of MRSA bacteraemias and the proportion of MRSA bacteraemias among all bacteraemias caused by $S$. aureus in Pirkanmaa HD and in TAUH have increased during the last decade (Table 1). The first

\section{TABLE 1}

Meticillin-resistant Staphylococcus aureus bacteraemias relative to all Staphylococcus aureus bacteraemias, in Tampere University Hospital, Pirkanmaa hospital district and Finland, Finland, 2001-2010

\begin{tabular}{|c|c|c|c|c|c|c|c|c|c|c|}
\hline \multirow[b]{2}{*}{ Years } & \multicolumn{10}{|c|}{ Number of MRSA bacteraemias/Number of all Staphylococcus aureus bacteraemias, (percentage) ${ }^{a}$} \\
\hline & 2001 & 2002 & 2003 & 2004 & 2005 & 2006 & 2007 & 2008 & 2009 & 2010 \\
\hline $\begin{array}{l}\text { Tampere University } \\
\text { Hospital }\end{array}$ & $\begin{array}{l}0 / 66 \\
(0 \%) \\
\end{array}$ & $\begin{array}{l}1 / 77 \\
(1 \%)\end{array}$ & $\begin{array}{l}3 / 75 \\
(4 \%)\end{array}$ & $\begin{array}{l}2 / 74 \\
(3 \%) \\
\end{array}$ & $\begin{array}{l}6 / 80 \\
(8 \%) \\
\end{array}$ & $\begin{array}{c}6 / 102 \\
(6 \%) \\
\end{array}$ & $\begin{array}{c}9 / 100 \\
(9 \%) \\
\end{array}$ & $\begin{array}{l}14 / 98 \\
(14 \%) \\
\end{array}$ & $\begin{array}{c}14 / 101 \\
(14 \%) \\
\end{array}$ & $\begin{array}{c}8 / 113 \\
(7 \%)\end{array}$ \\
\hline $\begin{array}{l}\text { Pirkanmaa Hospital } \\
\text { District }\end{array}$ & $\begin{array}{l}0 / 87 \\
(0 \%)\end{array}$ & $\begin{array}{l}2 / 92 \\
(2 \%)\end{array}$ & $\begin{array}{c}4 / 100 \\
(4 \%)\end{array}$ & $\begin{array}{c}5 / 100 \\
(5 \%)\end{array}$ & $\begin{array}{c}10 / 109 \\
(9 \%)\end{array}$ & $\begin{array}{c}9 / 130 \\
(7 \%)\end{array}$ & $\begin{array}{c}15 / 134 \\
(11 \%)\end{array}$ & $\begin{array}{c}16 / 120 \\
(13 \%)\end{array}$ & $\begin{array}{c}20 / 133 \\
(15 \%)\end{array}$ & $\begin{array}{c}11 / 150 \\
(7 \%)\end{array}$ \\
\hline Finland ${ }^{b}$ & $\begin{array}{c}4 / N R \\
(1 \%)\end{array}$ & $\begin{array}{l}9 / N R \\
(1 \%)\end{array}$ & $\begin{array}{c}7 / \mathrm{NR} \\
(1 \%)\end{array}$ & $\begin{array}{c}30 / N R \\
(3 \%)\end{array}$ & $\begin{array}{c}27 / N R \\
(3 \%)\end{array}$ & $\begin{array}{c}37 / N R \\
(3 \%)\end{array}$ & $\begin{array}{c}32 / \mathrm{NR} \\
(3 \%)\end{array}$ & $\begin{array}{c}40 / N R \\
(3 \%)\end{array}$ & $\begin{array}{c}30 / N R \\
(2 \%)\end{array}$ & $\begin{array}{c}26 / N R \\
(2 \%)\end{array}$ \\
\hline
\end{tabular}

MRSA: meticillin-resistant Staphylococcus aureus; NR: Not reported.

a A bacteraemia may have been reported more than once if the time between the bacteraemia episodes in a patient was $\geq 3$ months.

b Values for Finland are according to [7]. 
patient with MRSA bacteraemia in TAUH was diagnosed in October 2002.

\section{Characteristics of patients}

Baseline characteristics of patients with S. aureus bacteraemias included in the study are shown in Table 2. Fifty of 51 MSSA bacteraemia patients and 50 of 51 MRSA bacteraemia patients were Finnish. Of those with MRSA bacteraemia, 29 of 51 were known to be MRSA carriers prior to the bacteraemia episode. Forty four of the MRSA carriers had acquired MRSA from healthcare centres in the Pirkanmaa area while seven MRSA carrier cases were defined as community acquired. All patients with MRSA or MSSA bacteraemia were treated with an empirical antibiotic regimen, and when necessary antimicrobial treatment was changed according to culture results. Patients with MRSA bacteraemia were significantly older than those with MSSA bacteraemia, and had more severe underlying diseases (Table 2). Patients with MRSA bacteraemia were significantly more likely to have been transferred directly from another healthcare facility or were already in hospital upon bacteraemia onset ( $39 / 51$ vs $26 / 51, p=0.007$ ), and were more likely to have had treatment in some healthcare institution (hospital, long-term care, nursing home) during the preceding year than those with MSSA bacteraemia (47/48 vs 34/46, $p=0.001$, data available on 94 patients).

Disease severity did not differ between the two groups (Table 3).

In 45 of 51 of MRSA bacteraemia cases, MRSA strain type was spa type to67 (FIN-16), which is the epidemic strain type in Pirkanmaa HD [unpublished data]. Four of 51 were caused by spa type t172 (FIN-4) and two of 51 cases by to26/t4819 (FIN-10).

Nineteen of 51 MRSA bacteraemia patients and 22 of 51 of MSSA bacteraemia patients were in TAUH when bacteraemia was detected. Twenty-five of 51 MSSA bacteraemia patients and 12 of 51 MRSA bacteraemia patients were admitted to hospital from home, none of the MSSA patient and six of 51 MRSA patients from a long-term care institution, four of 51 MSSA patients and three of 51 MRSA patients from a healthcare centre ward, two of 51 MSSA patients and two of 51 MRSA patients from a regional hospital and none of the MSSA patients and nine of 51 MRSA patients from Tampere city hospitals.

\section{TABLE 2}

Characteristics and underlying conditions of patients with Staphylococcus aureus bacteraemia, Tampere University Hospital, Finland, October 2002-January $2010(n=102)$

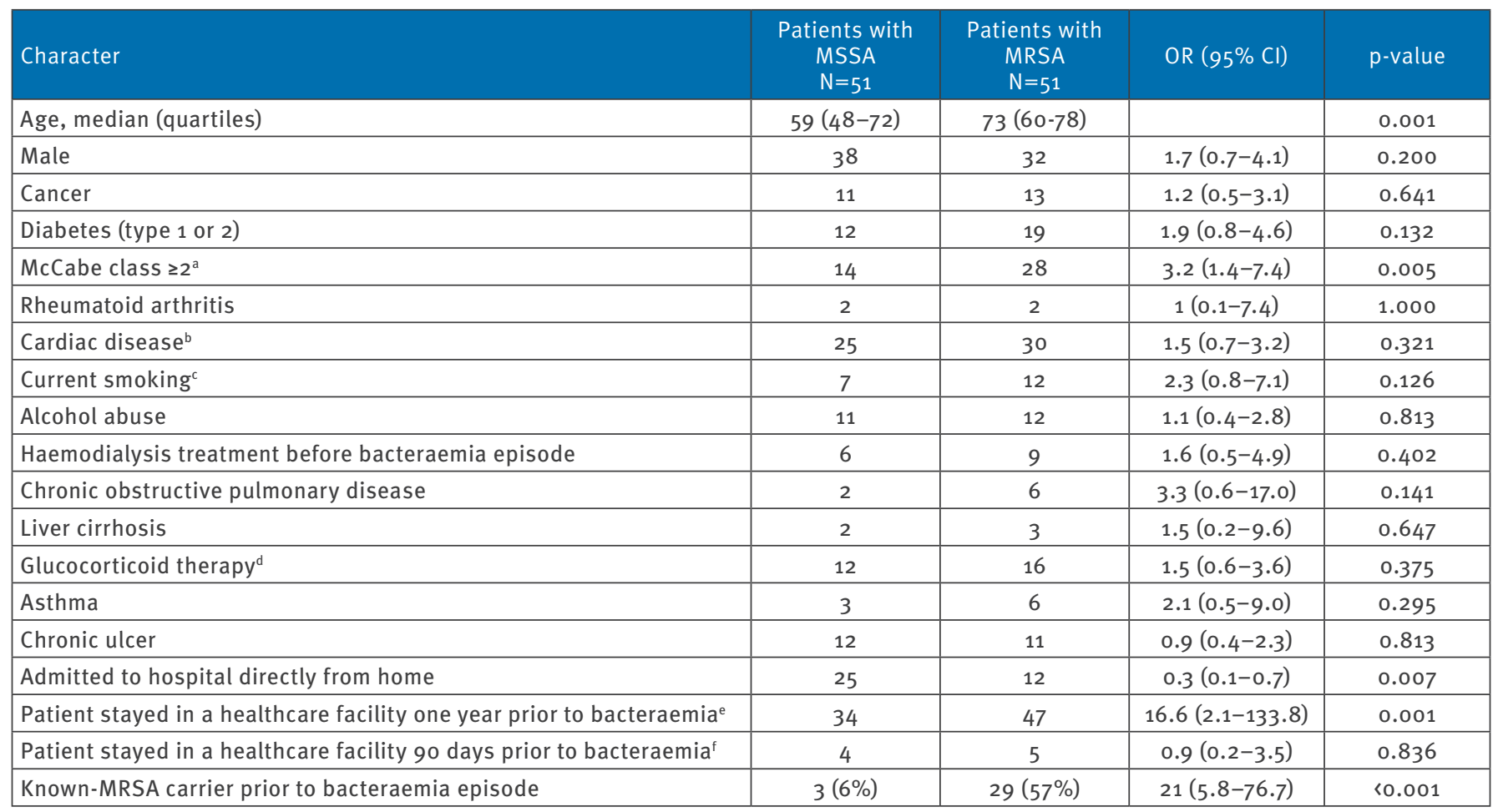

MSSA: meticillin-sensivite Staphylococcus aureus; MRSA: meticillin-resistant Staphylococcus aureus.

acCabe class $\geq 2$ : rapidly or ultimately fatal underlying disease.

${ }^{\text {b }}$ Coronary artery disease, valvular heart disease, cardiac myopathy or congestive heart failure.

c Data available for 64 patients.

${ }^{d} \geq 5 \mathrm{mg} /$ day of systemic prednisolone (or equivalent dose of another corticosteroid) during the preceding 30 days.

e Data available for 94 patients, 48 with MRSA and 46 with MSSA.

${ }^{f}$ Data available for 80 patients, 47 with MRSA and 33 with MSSA. 


\section{Antimicrobial treatment}

MRSA bacteraemia patients more seldom received adequate empirical antimicrobial therapy than those with MSSA bacteraemia (13/51 vs 43/51, p<0.001). Altogether 10 of 29 of those previously known to be MRSA carriers received inadequate empirical antimicrobial therapy. Ninety-eight of 100 patients in the study eventually received adequate antimicrobial treatment after susceptibility testing became available (data for changes in antibiotic treatment were missing for two MRSA cases, these patients having been transferred to regional hospitals). The delay until adequate antimicrobial treatment had a median o days (range: 0-10) in MSSA bacteraemia patients and a median two days (range: 0-5) in MRSA bacteraemia patients. Demographics or underlying diseases were not statistically significantly associated with the adequacy or inadequacy of antimicrobial treatment in MRSA bacteraemia (data not shown). The adequacy of treatment was not associated with the mode of admission (whether admission was direct from another hospital/healthcare facility or direct from home to hospital) (data not shown). Empirical antimicrobial therapy administered to patients is given in Table 4 .

\section{Discussion}

The present study shows that in Pirkanmaa HD, a notable percentage $(7-15 \%$ during recent years) of all $S$. aureus blood culture isolates are currently meticillin-resistant strains. The percentage of MRSA bacteraemias among all $S$. aureus bacteraemias in Pirkanmaa HD is high compared to corresponding figures for the whole of Finland. This may reflect the high rate of new MRSA notifications per year in our HD [7], or alternatively suggest that some regions in Finland, such as ours, are more affected by MRSA. Although Scandinavian countries including Finland have been considered low prevalence countries, substantial regional variations are common $[7,10]$.

\section{TABLE 3}

Case fatality, sources of bacteraemia and adequacy of treatment in patients with Staphylococcus aureus bacteraemia, Tampere University Hospital, Finland, October 2002-January $2010(n=102)$

\begin{tabular}{|l|c|c|c|c|}
\hline Character & $\begin{array}{c}\text { MSSA } \\
\mathrm{N}=51\end{array}$ & $\begin{array}{c}\text { MRSA } \\
\text { patients } \\
\mathrm{N}=51\end{array}$ & OR (95\% Cl) & $p$-value \\
\hline Case fatality & 11 & 12 & $1.1(0.4-2.8)$ & 0.813 \\
\hline $\begin{array}{l}\text { Empirical treatment } \\
\text { adequate }\end{array}$ & 43 & 13 & $0.1(0.0-0.2)$ & $<0.001$ \\
\hline Focus known & 38 & 42 & $1.6(0.6-4.2)$ & 0.336 \\
\hline Skin & 20 & 17 & $0.8(0.3-1.7)$ & 0.537 \\
\hline Osteomyelitis & 10 & 4 & $0.3(0.1-1.2)$ & 0.084 \\
\hline Arthritis & 3 & 3 & $1.0(0.2-5.2)$ & 1.000 \\
\hline Endocarditis & 3 & 0 & $0.5(0.4-0.6)$ & 0.243 \\
\hline Catheter-related & 11 & 14 & $1.4(0.6-3.4)$ & 0.490 \\
\hline Device infection & 4 & 12 & $3.6(1.1-12.1)$ & 0.029 \\
\hline
\end{tabular}

a The case fatality rate was studied within 30 days after a positive blood culture.
By retrospectively reviewing the hospital records of 102 patients with $S$. aureus bacteraemias, who had been admitted to TAUH from October 2002 to January 2010, we found that patients with MRSA bacteraemia were significantly older than those with MSSA and/or had higher McCabe class and/or had previous MRSA carrier status. In accordance with previous reports [2] comorbidities and healthcare facility stay were significant factors associated with the risk of MRSA bacteraemia. Interestingly, the risk factors for MRSA bacteraemia in our study did not differ from the risk factors reported in counties where MRSA is endemic [3,4]. Most of MRSA bacteraemia cases in the present study were caused by the spa type to67 (FIN-16), which has caused a wide ongoing epidemic in Pirkanmaa HD since 2001.

MRSA bacteraemia was associated with an increased risk of inadequate empirical antimicrobial therapy. Inadequate empirical therapy was common also for patients previously known to be MRSA carriers. Despite a low statistical power due to a low number of patients, the present study is in line with the findings of a recent multicentre study in nine western European countries indicating that empirical antimicrobial treatment frequently fails in patients with MRSA bacteraemia [10]. Cefuroxime, which is not effective in MRSA bacteraemia, has been a widely used empirical regimen among patients with community-acquired bacteraemia in Finland [11]. It has been used to treat patients with common infections such as skin and soft tissue infections. Intravenous cefuroxime was by far the most common agent chosen as an empirical antibiotic therapy in S. aureus bacteraemia in this study. The present study indicates that local guidelines for empirical antimicrobial therapy should be updated for MRSA endemicity, guidelines should be made available on the internet and doctors should be widely informed on the new guidelines to be published. This may be done for example in the form of an electronic bulletin. MRSA is a potential cause of severe infections in MRSA carriers. Vancomycin is now recommended empirically for all previously known MRSA carriers with suspected or documented sepsis or other severe infection or infection potentially caused by S. aureus (i.e. osteomyelitis,

\section{TABLE 4}

Empirical antimicrobial treatment in patients with Staphylococcus aureus bacteraemia, Tampere University Hospital, Finland, October 2002-January 2010 (n=102)

\begin{tabular}{|l|c|c|}
\hline Empirical treatment & $\begin{array}{c}\text { MRSA bacteraemia } \\
(\mathrm{n}=51)\end{array}$ & $\begin{array}{c}\text { MSSA bacteraemia } \\
(\mathrm{n}=51)\end{array}$ \\
\hline Cephalosporins & 29 & 38 \\
\hline Vancomycin & 8 & 2 \\
\hline Piperazillin-tazobactam & 8 & 1 \\
\hline Clindamycin & 2 & 4 \\
\hline Linezolid & 4 & 0 \\
\hline Daptomycin & 1 & 0 \\
\hline
\end{tabular}

MRSA: meticillin-resistant Staphylococcus aureus; MSSA: meticillin-sensitive Staphylococcus aureus. 
endocarditis, post-surgery infections) in Pirkanmaa HD. Moreover, although the importance of administering an antimicrobial agent with good activity against MRSA to MRSA carriers has been highlighted in hospital hygiene education of staff in our institution, this may not be sufficient. Information regarding a previous MRSA finding has appeared in patients' electronic hospital records for many years. This information has been made more conspicuous during the last years, as an electronic alert system for MRSA has been constructed for hospital records.

Novel molecular methods may offer some advance in reducing the time frame for detecting mecA gene, the major determinant for meticillin resistance in $S$. aureus, in patients with $S$. aureus bacteraemia. These methods may be used to guide empiric antimicrobial therapy before definite culture-based susceptibility testing is available [12]. Also, in the case of bacteraemia in a MRSA carrier, molecular methods for the detection of mecA gene are now adapted for clinical use to speed up microbiological diagnosis.

Some limitations must be conceded here. There were altogether 92 patients with MRSA bacteraemias in Pirkanmaa HD during the period 2001 to January 2010 (Table 1), most of these patients having been treated in TAUH. However, we did not study the hospital records of patients treated in other hospitals than TAUH. Only one bacteraemia episode (the first) per patient was included in the study and patients with polymicrobial bacteraemias were excluded. As the study was retrospective by design, the data regarding socioeconomic status and travel history were not acquirable. The data regarding the number of days of hospital prior to bacteraemia and data regarding a contact with a known MRSA carrier could not be obtained. Although not done in the present study, the use of total MSSA bacteraemia patients (2001-2010) as a control group would have increased the statistical power of this study.

The present study is a case-control study with regard to the assessment of risk factors for meticillin resistance. The study was not designed to assess comparative outcomes, effects of appropriate vs. inappropriate empirical treatment or risk factors for death. The risk factors for meticillin resistance were studied because the case-control patients here were not matched for age, sex or other characteristics. Some work indicates that advanced age would be a significant factor associated with the adequacy of empirical therapy in MRSA bacteraemia [13], but this was not confirmed in the present study.

\section{Acknowledgments}

We thank Mrs Anneli Keinonen and hygiene control nurse Jaana Sinkkonen for excellent technical assistance. This study was financially supported by the Competitive Research Funding of the Tampere University Hospital.

\section{References}

1. Liu C, Bayer A, Cosgrove SE, Daum RS, Fridkin SK, Gorwitz RJ, et al. Clinical practice guidelines by the infectious diseases society of america for the treatment of methicillin-resistant Staphylococcus aureus infections in adults and children: executive summary. Clin Infect Dis. 2011;52(3):285-92.

2. Viallon A, Marjollet O, Berthelot P, Carricajo A, Guyomarc'h $\mathrm{S}$, Robert F, et al. Risk factors associated with methicillinresistant Staphylococcus aureus infection in patients admitted to the ED. Am J Emerg Med. 2007;25(8):880-6.

3. Shurland S, Zhan M, Bradham DD, Roghmann MC. Comparison of mortality risk associated with bacteremia due to methicillinresistant and methicillin-susceptible Staphylococcus aureus. Infect Control Hosp Epidemiol. 2007;28(3):273-9.

4. Cosgrove SE, Sakoulas G, Perencevich EN, Schwaber MJ, Karchmer AW, Carmeli Y. Comparison of mortality associated with methicillin-resistant and methicillin-susceptible Staphylococcus aureus bacteremia: a meta-analysis. Clin Infect Dis. 2003;36(1): 53-9.

5. Kerttula AM, Lyytikäinen 0 , Kardén-Lilja M, Ibrahem $S$, Salmenlinna S, Virolainen A, et al. Nationwide trends in molecular epidemiology of methicillin-resistant Staphylococcus aureus, Finland, 1997-2004. BMC Infect Dis. 2007;7:94.

6. Stenhem $M$, Ortqvist $A$, Ringberg $H$, Larsson L, Olsson Liljequist B, Haeggman S, et al. Imported methicillinresistant Staphylococcus aureus, Sweden. Emerg Infect Dis. 2010;16(2):189-96.

7. Hulkko T, Lyytikäinen O, Kuusi M, Seppälä S,Ruutu P. Infectious Diseases in Finland 1995-2009. Finnish National Institute for Health and Welfare (THL). Helsinki: THL. 2010. [Accessed 01 Sep 2011]. Available from: http://www.thl.fi/ thl-client/pdfs/d6d63c66-9690-4f4d-9ee1-319bb5648eaf

8. Statistics Finland. [Accessed 24 Jul 2011]. Available from: http://www.stat.fi/

9. McCabe WR, Jackson GG. Gram-negative bacteremia. II. Clinical, laboratory, and therapeutic observations. Arch Intern Med. 1962;110(6): 856-64.

10. Ammerlaan H, Seifert H, Harbarth S, Brun-Buisson C, Torres A, Antonelli M, et al. Adequacy of antimicrobial treatment and outcome of Staphylococcus aureus bacteremia in 9 Western European countries. Clin Infect Dis. 2009;49(7):997-1005.

11. Arstila T, Auvinen $\mathrm{H}$, Huovinen P. Beta-lactam resistance among Escherichia coli and Klebsiella species blood culture isolates in Finnish hospitals. Finnish Study Group for Antimicrobial Resistance. Eur J Clin Microbiol Infect Dis. 1994;13(6):468-74.

12. Paolucci M, Landini MP, Sambri V. Conventional and molecular techniques for the early diagnosis of bacteraemia. Int J Antimicrob Agents. 2010;36 Suppl 2: S6-16.

13. Marchaim D, Kaye KS, Fowler VG, Anderson DJ, Chawla V, Golan Y, et al. Case-control study to identify factors associated with mortality among patients with methicillin-resistant Staphylococcus aureus bacteraemia. Clin Microbiol Infect. 2010;16(6):747-52. 